\title{
IMAGEN MENTAL REPRODUCTORA ESTÁTICA Y CINÉTICA EN SUJETOS CON RETARDO MENTAL LEVE Y MODERADO
}

\author{
MENTAL IMAGE REPRODUCTIVE STATIC AND KINETIC IN SUBJECTS WITH \\ MILD AND MODERATE MENTAL RETARDATION
}

\author{
José Carlos Rivera Benavides ${ }^{1}$ \\ Universidad Nacional Mayor de San Marcos, Lima, Perú \\ (Recibido el 18/08/2011, Aceptado el 02/12/2011)
}

\begin{abstract}
RESUMEN
En la psicología genética de J. Piaget, la imagen mental marca el inicio de las funciones cognitivas representacionales figurales (2-7 años); junto a B. Inhelder estudiaron las imágenes espaciales reproductoras dentro de las cuales, las más elementales son las estáticas y cinéticas. Asumimos que ellas están implicadas en la inteligencia ejecutiva. De aquí nuestro propósito de explorarlas en sujetos con retardo mental leve y moderado, quienes no alcanzan el dominio de la representación operatoria. Para el efecto aplicamos la Escala Ejecutiva de Borelli Oléron, la cual reformulamos en términos de constructos de imágenes estáticas y cinéticas. Los resultados encontrados fueron que, los sujetos con retardo mental leve rindieron más en las imágenes estáticas y más aún en las cinéticas en comparación con los moderados.
\end{abstract}

Palabras Clave: funciones cognitivas representacionales figurales; imagen mental reproductora estática y cinética; inteligencia ejecutiva; retardo mental leve y moderado.

\begin{abstract}
ABSTRAC
In genetic psychology of J. Piaget, the mental image marks the beginning of representational figural cognitive functions (2-7 years) with B. Inhelder studied the breeding space images within which the most elementary are the static and kinetic. We assume that they are involved in executive intelligence. Here our intention to explore in subjects with mild to moderate mental retardation who do not reach the operative domain representation. For this purpose we apply the Scale Executive Borelli- Oléron, which the reformulated in terms of constructs of static and kinetic images.

The results were that subjects with mild mental retardation yielded more static images and even more in the kinetics compared to the moderates.

Keywords: figural representational cognitive functions; reproductive mental image static and kinetic, executive intelligence, mild to moderate mental retardation.
\end{abstract}

1 Docente Asociado de la Facultad de Psicología de la Universidad Nacional Mayor de San Marcos

E-mail: jocarivera@gmail.com 


\section{INTRODUCCIÓN}

En la teoría psicogenética de Jean Piaget (Pain, 1974) los procesos cognitivos se clasifican en figurales y operatorios. La función representativa se organiza con los procesos figurales, principalmente con la imagen mental, la que está relacionada con los acontecimientos espaciales (Laurendeau y Pinard, 1976) estáticos y cinéticos que el niño logra representarlos mediante reproducción imitativa internalizadas en la etapa del sub-estadío I: aparición de la función simbólica y principio de la representación (2-4 años). El niño se comportará con las imágenes de la misma manera que con los objetos en el estadío sensomotor. (Piaget et Inhelder, 1966).

La imagen mental es psicogenéticamente anterior a las operaciones concretas, y estas las subsumen luego como significantes de los estados de objetos y acontecimientos que lo rodean, incluyendo su propio cuerpo (Pinol-Douriez, 1979). La imagen, pues, se encuentra ligada a las acciones intelectuales de carácter espacial.

Conocer si los sujetos con retardo mental tienen la capacidad de manejar imágenes elementales como las de reproducción estática y cinética nos animó a la presente investigación.

La adaptación intelectual de los sujetos con Retardo Mental depende, como es obvio, de una serie de factores entre los cuales podemos destacar los biopsicológicos y las deprivaciones sociales, hasta los trastornos emocionales y psicopatológicos. De una $u$ otra manera éstos factores están ligados a los procesos cognitivos de la inteligencia abstracta como el pensamiento y el lenguaje pero también, a la inteligencia figurativa que es más temprana (entre los $2-6$ años). Entre ellos destacamos el rol de la imagen mental que como actividad imitativa, interiorizada e interpretativa, permite la reproducción presente o evocada de acontecimientos físico-espaciales y, como actividad anticipatoria permite la planificación de coordinaciones previsoras de los probables acontecimientos físicos-espaciales.

Poseer imágenes topográficas de su entorno (Piaget et Inhelder, 1972), nociones de simetría y equilibrio en el manejo del cuerpo y de juegos constructivos, transposición de un código de figuras planas a uno tridimensional o viceversa, constituyen capacidades que influirán en la adquisición de habilidades para acercarse a la comprensión del orden geométrico del espacio, mejorando su percepción de las cosas y su representación de ellas, sin lo cual le será difícil asimilar, incluso, nociones elementales de clases y conjuntos; en resumen de una buena iniciación en las operaciones matemáticas básicas.

Desde este punto de vista se podrían realizar estudios más detallados del papel de la imagen en el aprendizaje de los fenómenos físicos y las formas espaciales, aspectos sobre los que he observado no se tienen en cuenta en los programas educativos.

Los estudios sobre el razonamiento en los débiles mentales (Inhelder, 1971), antecedentes en el sentido de una relación empírica sobre el tema de tesis, más bien constituye un marco dentro de la teoría general de la psicología genética, pues el razonamiento operatorio tiene relación con la imagen mental en el proceso del desarrollo cognitivo, por lo que recurrimos a esta fuente, además de no haber 
encontrado trabajos similares al presente estudio. Lo que ocurre en el nivel del razonamiento también sucede en la imagen mental puesto que ambos se basan en los mecanismos cognitivos figurales y operatorios.

Así, las Imágenes Reproductoras Estáticas y Cinéticas empiezan a ser logradas con mayor facilidad a los 4-7 años aproximadamente, tratándose de las posiciones de los objetos en una reproducción inmediata o diferida, no ocurriendo lo mismo con las Imágenes Anticipadoras y de Transformación que están conectadas con la constitución de las operaciones concretas de carácter espacial. (Piaget e Inhelder, 1973, pp. 100-107).

De esta manera nuestro objetivo general de estudio es determinar la diferencia en la elaboración de la Imagen Mental Reproductora Estática vs. Cinética entre sujetos con Retardo Mental Leve vs. Moderado en una Escala de Inteligencia Ejecutiva (Borelli y Oléron, 1964/1995), según género y edad cronológica.

\section{MÉTODO}

\section{Diseño}

Originalmente planteado como un estudio exploratorio (Rivera, 1995) arribamos a uno de tipo correlacional con el respectivo diseño transeccional correlacional.

En 2003 aplicamos la escala Borelli-Oléron en niños y jóvenes con Retardo Mental pero no encontramos diferencias importantes ni por el sexo ni por la edad cronológica, a esta etapa corresponde nuestra primera hipótesis de investigación (H1) referida a la cualidad discriminativa genética de la escala. En 2008, a la luz de la teoría de la imagen mental hicimos un análisis de contenido de cada una de las 7 Pruebas de la Escala Ejecutiva de Borelli-Oléron y, por validez de juicio hipotetizamos acerca de la existencia de dimensiones diferentes de la Imagen Mental Reproductora Estática y Cinética en cada una de las Pruebas de la Escala, que al ser contrastadas favorablemente por las correlaciones entre ellas, logramos avanzar con nuestra segunda hipótesis de investigación (H2).

Finalmente, obtuvimos los resultados de la aplicación del Borelli-Oléron, en sujetos diferenciados por el tipo de Retardo Mental, Leves Vs. Moderados en las Imágenes Mentales Reproductoras, Estáticas Vs. Cinéticas, correspondiente a nuestra tercera hipótesis de investigación (H3).

\section{Participantes}

En este estudio sobre imagen mental en sujetos con retardo mental leve (RML) y moderado (RMM) usamos un muestreo intencional de sujetos con esas características, en Centros de Educación Especial de la ciudad de Lima, del Ministerio de Educación del Perú.

La muestra de esta población se obtuvo de dos Centros Educativos Básicos Especiales: CEBE Beatriz Cisneros y CEBE ${ }^{\circ}$ 6: 40 hombres y 40 mujeres. De tal modo que $\mathrm{N}=80$ sujetos, tamaño definitivo de la muestra, con edades 
comprendidas entre los 12 y 20 años, de tal modo que por edad existen por lo menos tres sujetos (registros) en cada sub-muestra por sexo (cantidad mínima necesaria para la obtención de los baremos), habiendo grupos de edad conformados por cuatro, cinco y hasta seis sujetos.

Tabla 1. Sujetos con Retardo Mental agrupados según Edad y Sexo

\begin{tabular}{c|c|c|c}
\hline & \multicolumn{3}{c}{ SEXO } \\
\cline { 2 - 3 } EDADES & HOMBRES & MUJERES & TOTALES \\
\hline 12 & 6 & 3 & 9 \\
13 & 5 & 4 & 9 \\
14 & 5 & 4 & 9 \\
15 & 4 & 4 & 8 \\
16 & 6 & 6 & 12 \\
17 & 4 & 5 & 9 \\
18 & 4 & 4 & 8 \\
19 & 3 & 5 & 8 \\
20 & 3 & 5 & 8 \\
TOTALES & 40 & 40 & 80 \\
\hline
\end{tabular}

Dado que nuestro interés era explorar los mecanismos de la imagen mental, la cual es una función cognitiva de carácter figural estrechamente ligada al desarrollo de la inteligencia ejecutiva y en particular a la adquisición de las nociones espaciales y dado que la imagen mental tiene un carácter psicogenético, reagrupamos a los sujetos por tipo de retardo y su rendimiento en la escala Borelli-Oléron.

Tabla 2. Sujetos agrupados según Sexo y tipo de Retardo Mental

\begin{tabular}{c|c|c|c}
\hline & \multicolumn{2}{c}{ TIPO DE RETARDO MENTAL } & \multicolumn{1}{c}{ TOTALES } \\
\cline { 2 - 3 } SEXO & RML & RMM & 39 \\
\hline HOMBRES & 19 & 20 & 41 \\
MUJERES & 21 & 20 & 80 \\
TOTALES & 40 & 40 & \\
\hline
\end{tabular}

De otro lado hay que señalar que según el DSM-IV (Manual Diagnóstico y Estadístico de los Trastornos Mentales) el Retraso Mental Leve con frecuencia no es distinguible de otros niños sin retraso mental hasta edades posteriores (después de los 5-6 años de edad).

En el caso del retardo mental moderado estos constituyen un grupo que en general no progresan más allá de un segundo nivel en materias escolares y la repitencia también puede hacerlos no distinguibles en relación a su edad escolar. 
En ambos grupos son las condiciones de repitencia y fracaso escolar, acompañados de criterios clínicos los que deciden su traslado a Centros de Educación Especial, pero en nuestro medio no existen procedimientos longitudinales estándar para la determinación de la evolución intelectual en niños de 0-10 años aproximadamente, que nos permite el diagnóstico y la intervención psicopedagógica adecuadas.

\section{Variables de Estudio}

- $\quad$ Imagen mental reproductora: estáticas/cinéticas.

- Inteligencia ejecutiva: rendimiento en la escala ejecutiva de Borelli-Oléron.

- $\quad$ Retardo mental: rendimiento en el Wais (CI) que clasifica a los sujetos con RML y RMM.

- $\quad$ Sexo: hombre/mujer.

- $\quad$ Edad: grupos de 12 a 20 años.

\section{Instrumentos de Recolección de Datos}

Para la recolección de la data se utilizó la Escala Ejecutiva de Borelli-Oléron, conformada por 7 Pruebas de Inteligencia Ejecutiva y Desarrollo, además probadamente útil para sujetos en los que la comunicación verbal deficitaria no permite apreciar con claridad su nivel de desarrollo intelectual.

Las pruebas ejecutivas requieren para su solución un conjunto de habilidades intelectuales aprendidas. El nombre de Ejecutiva viene del hecho cuando un sujeto intenta responder a ella no lo hace mediante categorías verbales predominantemente. "Las Pruebas o Escalas Psicológicas no verbales prescinden de todo elemento verbal (oral o escrito) en su material de examen propiamente dicho" (Tavella, 1966, p. 122). Las instrucciones son no lingüísticas lo que llevó a los psicólogos a elaborar pruebas que midieran la inteligencia general menos sensibles a las influencias culturales y al factor específico verbal, pero no a la ausencia de signos en términos absolutos, pues ellas requieren de procesamientos figurativos. Tavella, divide las pruebas no verbales en tests gráficos y de ejecución o performance tests (Op.cit, pp.122-123).

Piaget nos habla de Imagen Mental como un proceso que supera las leyes de la percepción y es el soporte del simbolismo por imitación directa o diferida (Piaget, 1961 y 1975). ¿Qué nos lleva a suponer que las Escalas Ejecutivas requieren de la función de la imagen? Primero, toda vez que la particularidad física de un objeto se enmarca o adquiere la cualidad de un percepto, ella es susceptible de ser utilizada, para representar por imitación directa o diferida una acción imaginada, por ejemplo: tomar la tapa de una olla (figura perceptual) y simular el timón de un auto (acción imaginada o acción guiada por una imagen), en segundo lugar, y en particular, la definición de reproducción diferida de acuerdo al Cuadro 3 es la respuesta típica que da el sujeto a los problemas que plantea la Escala BorelliOléron sea la reproducción inmediata (I) o diferida (II). 
Tabla 3. Clasificación de las Imágenes Mentales

\begin{tabular}{l|c|c}
\multicolumn{1}{c}{ IMAGENES } & $\begin{array}{c}\text { INMEDIATAS } \\
\text { (I: pre-imágenes o } \\
\text { diferidas II) }\end{array}$ & $\begin{array}{c}\text { Que tratan sobre el Producto } \\
\text { (P) o sobre la modificación } \\
\text { (M) }\end{array}$ \\
\hline $\begin{array}{l}\text { - Reproductoras (R): } \\
\text { Estáticas (RE) }\end{array}$ & RTP o RTM \\
Cinéticas (RC) & REI o REII & RTP o RTM \\
De Transformación (RT) & RCI o RCII & \\
- Anticipadoras (A): & RTI o RTII & ACP o ACM \\
Cinéticas (AC) & & ATP o ATM \\
De Transformación (AT). & & \\
\hline
\end{tabular}

Fuente. Tomado de Piaget et Inhelder (1966:16)

\section{Análisis de Contenido de la Escala Borelli-Oléron}

Las acciones sensomotoras son una condición necesaria para la manipulación de objetos, instrumentos, y en fin para llegar a establecer relaciones medios-fin de carácter exploratorio y compositivo que observamos desde que el niño pequeño "amontona" una heterogeneidad de cosas hasta que realiza clasificaciones de objetos según ciertas magnitudes. Entre uno y otro caso hay un recorrido de experiencias físicas sobre las cuales el sujeto construye esquemas de acción. Un ejemplo de esquema es la agrupación simétrica de un conjunto de objetos que da como resultado una figura sin más significado que el equilibrio y/o ensamble entre ellos, sujetos a las condiciones gravitatorias y de espacio en la que el sujeto aplica movimientos espontáneamente - todavía sin estrategias - y produce escenas que al ser percibidas visualmente pueden encajar en una gestalt reconocible total o parcialmente. Estos procesos son típicos del juego simbólico en sus inicios durante el cual el sujeto explora el comportamiento de las propiedades de los objetos, comportamiento que obedece a leyes físicas espaciales. Luego accederá a manipular los objetos con el fin de obtener configuraciones equilibradas y con cierta disposición espacial, utilizando esta vez estrategias que constituyen un equilibrio entre la acomodación y la asimilación, o sea un esquema de acción consolidado, pues no hay pruebas de ensayo y error, o exploración, de los objetos, que dan lugar a gestalts casuales, sino que por el contrario busca que reproducirlas y transformarlas por lo que la imagen no es la prolongación de la percepción como tal, sino la de la actividad perceptiva y entonces la imagen se constituye en el símbolo de la acción, que consiste en constituir una figura (siguiendo los contornos del objeto, etc.), o bien en transformarla. Constitución y transformación de una figura son los hechos fenómenos físico-espaciales en el que la constitución tiende a un estado estático y la transformación a un estado dinámico. Hay que puntualizar que las transformaciones 
comprenden los desplazamientos de los objetos por traslación y rotación (diferentes de las transformaciones operatorias como en el caso de la sustancia, el peso y el volumen). Daremos dos ejemplos:

La tarea consiste en encajar figuras geométricas, bajo la siguiente instrucción, "pon estas piezas aquí" (a, b, c y d sucesivamente) según la siguiente figura:

a
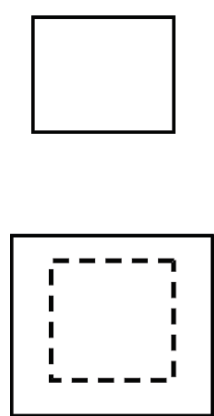

$\mathrm{b}$
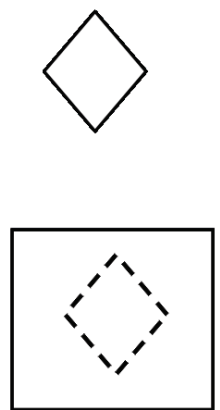

c
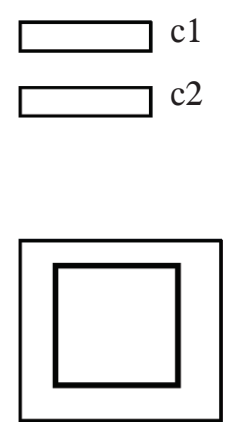

d
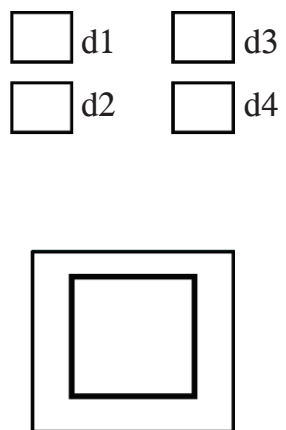

Figura 1. Encaje de Figuras Geométricas por Reproducción Estática. Elaboración propia.

La realización de esta tarea requiere de una traslación directa de las piezas al encaje. Este procedimiento se basa en la correspondencia por centración perceptiva, sobre todo en a y b. En c y d, el encaje consiste en una relación de partes en un todo, pero igual se mantienen las traslaciones por centración. Las traslaciones en a y $\mathrm{b}$ y en c y d, se basan en la noción topológica de vecindad. Por ejemplo c1 coincide con un sector del cuadrado de encaje (la recta paralela y los ángulos de c, con la parte superior o inferior del cuadrado de encaje, c2 entonces es una figura idéntica a la restante porción del cuadrado de encaje y entonces el sujeto completa la tarea.

2) Según la misma instrucción que en 1)

a

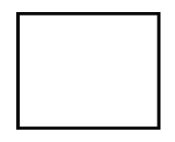

b
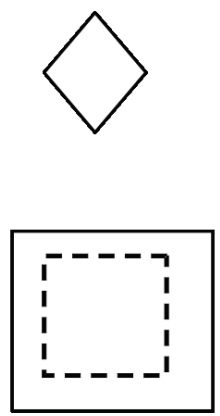

c
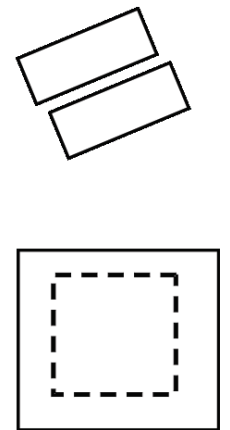

d
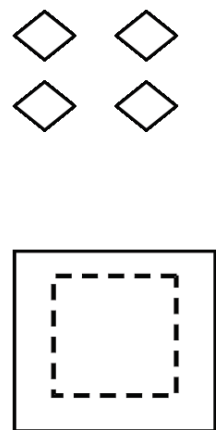

Figura 2. Encaje de Figuras Geométricas por Reproducción Cinética. Elaboración propia. 
La tarea en este caso requiere de traslación y rotación de las piezas, para compensar la descentración, además de la relación partitiva con todo y la relación de vecindad como en el ejemplo 1.

En conclusión diremos que en el ejemplo 1 hay traslación mediante el movimiento aplicado por el sujeto, sin que la figura varíe su identidad, mientras en el ejemplo 2 la identidad varía y para realizar el encaje se tienen necesariamente que hacer rotaciones para lograr el encaje. Esto prueba que la imagen no es la prolongación de la percepción como tal, sino de la actividad perceptiva. Así la centración es dominante en el ejemplo 1 y la descentración lo es en el ejemplo 2 .

La Escala Borelli-Oléron (Escala B-O) se rige por estos procesos, es decir, encontramos actividades perceptuales que generan imágenes de identidad. El sujeto construye o reproduce a' mediante a y logra que $\mathrm{a}=\mathrm{a}$ '. Mediante esta Escala nos hemos interesado en transformaciones mucho más elementales, transformaciones que no afectan a la forma del objeto sino, únicamente, a su situación, posición, desplazamientos, en suma, a su situación en el espacio. Con estos criterios formulamos el Análisis de Contenido de la Escala, prueba por prueba, como sigue a continuación y, cuyo resultado presentamos en el Cuadro 4 .

Prueba I: Es un tipo de Imagen de Reproducción Estática Inmediata (REI) ya que el sujeto tiene que reconstruir modelos idénticos después de 5 segundos de exposición de los modelos originales. Éstos son presentados en tarjetas y el sujeto traslada a cubos tridimensionales la composición gráfica vista en las tarjetas.

Prueba II: Es un tipo de Imagen de Reproducción Estática Inmediata (REI) ya que el sujeto debe armar el cuerpo entero de un maniquí cuyas piezas representan los miembros de manera invertida y bajo su mirada.

Prueba III: Plancha A. Imagen de Reproducción Cinética Inmediata (RCI). La tarea es encajar dos rectángulos en un cuadrado. Éstos se presentan bajo la vista del sujeto y en desorden. El sujeto tiene que hacer uso de giros de las piezas para lograr el encaje.

Plancha $B$ y $C$. Imagen de Reproducción Cinética Inmediata (RCI). Del mismo modo de ejecución, pero esta vez con dos planchas para el encaje (B y C) con variación de las figuras para encajar (triángulos, cruz, etc.) y donde las piezas son pequeñas, grandes y en mayor número. La tarea se realiza simultáneamente en B y C.

Prueba IV: Imagen de Reproducción Cinética Inmediata (RCI). Comparado con la Prueba III tanto el encaje como las piezas a encajar son polígonos de cuatro lados y diferentes tamaños. En este caso la prueba se repite 3 veces. En cada ensayo el tiempo debe ser menor.

Prueba V: Imagen de Reproducción Cinética Inmediata (RCI). Describimos esta prueba como tal dado que el sujeto tiene como tarea reproducir inmediatamente después de haber visto cómo el experimentador realiza movimientos acompasados en una serie de órdenes alternadas que va golpeando frente al sujeto quien tiene 
que repetir los movimientos hechos por el experimentador, inmediatamente.

Prueba VI: Imagen de Reproducción Cinética Inmediata (RCI). La tarea del sujeto consiste en copiar con un lápiz diversas formas geométricas presentadas en una hoja modelo.

Prueba VII: Imagen de Reproducción Estática Inmediata (REI). Describimos esta prueba en esta categoría porque los movimientos que realiza el sujeto para reproducir en forma tridimensional requieren de movimientos que culminan en la dirección de las perspectivas de las tarjetas, por un procedimiento de copia directa.

Tabla 4. Imagen Mental Reproductora en las Pruebas de la Escala B-O

\begin{tabular}{c|c}
\hline $\begin{array}{c}\text { TIPOS DE IMAGEN } \\
\text { MENTAL }\end{array}$ & $\begin{array}{c}\text { PRUEBAS DE LA ESCALA } \\
\text { BORELLI - OLERON }\end{array}$ \\
\hline Reproductora Estática & I, II, VII \\
Reproductora Cinética & III, IV, V, VI \\
\hline
\end{tabular}

\section{CONFIABILIDAD Y VALIDEZ}

Según David Magnusson (1983), la teoría de la confiabilidad básica se basa en la definición dada de confiabilidad como la correlación entre test paralelos, pues no es necesario repetir las medidas con el mismo instrumento para estimar su confiabilidad.

En el presente trabajo hemos recurrido a la medida del CI como una variable muestral (o de criterio) de los sujetos de estudio (Retardo Mental Leve y Moderado) a quienes se les aplicó la Escala Ejecutiva Borelli-Oléron, la que se dimensionó en Imágenes Estáticas y Cinéticas para la construcción de la hipótesis básica del estudio (Hipótesis 2). Pero como ambas pruebas comparten el factor ejecutivo de la inteligencia, no constituyendo un cien por cien de paralelismo, entonces procedimos a medir la correlación entre Escala B-O y CI, para esto acudimos a la validez concurrente.

En este caso se dispone de la medida de la variable de criterio en el momento en que se obtienen los resultados del test. El diagnóstico clínico de la lesión cerebral, sobre el cual estuvieron de acuerdo varios neurólogos, puede usarse, por ejemplo, como criterio para la validez de un test de lesión cerebral (...) cuando computamos los coeficientes de validez, es necesario que los datos del test y los de criterio se determinen en forma independiente [para que el psicólogo no sea] presa de lo que se conoce como contaminación de criterio. (Magnusson, 1983, p. 158-160).

En nuestro caso el CI es la medida de criterio, o sea el referente para establecer la validez de la Escala B-O, cuyos resultados se pueden ver en la siguiente Tabla: 
Tabla 5. Matriz de Coeficiente de Correlación de Pearson entre los resultados del CI, las Pruebas de la Escala Borelli-Oléron, el total en la Escala y sus respectivos totales en Imagen Mental Estática y Cinética.

\begin{tabular}{l|l|l|l|l|l|l|l|l|l|l|l}
\hline CI & \multicolumn{1}{c}{ P1 } & P2 & \multicolumn{1}{c}{ P3 } & \multicolumn{1}{c}{ P4 } & P5 & \multicolumn{1}{c}{ P6 } & \multicolumn{1}{c|}{ P7 } & \multicolumn{1}{c}{ TOTAL Estática Cinética } & \\
\hline CI & 1.00 & & & & & & & & & & \\
P1 & 0.41 & 1.00 & & & & & & & & & \\
P2 & 0.20 & 0.38 & 1.00 & & & & & & & & \\
P3 & 0.42 & 0.43 & 0.22 & 1.00 & & & & & & & \\
P4 & 0.36 & 0.24 & 0.17 & 0.51 & 1.00 & & & & & & \\
P5 & 0.40 & 0.46 & 0.11 & 0.26 & 0.27 & 1.00 & & & & & \\
P6 & 0.52 & 0.44 & 0.20 & 0.44 & 0.53 & 0.46 & 1.00 & & & & \\
P7 & 0.34 & 0.44 & 0.27 & 0.46 & 0.37 & 0.42 & 0.52 & 1.00 & & & \\
TOTAL & 0.57 & 0.74 & 0.40 & 0.73 & 0.67 & 0.65 & 0.78 & 0.71 & 1.00 & & \\
Estática & 0.44 & 0.90 & 0.58 & 0.51 & 0.34 & 0.48 & 0.53 & 0.73 & 0.84 & 1.00 & \\
Cinética & 0.57 & 0.53 & 0.23 & 0.75 & 0.77 & 0.66 & 0.81 & 0.60 & 0.95 & 0.62 & 1.00 \\
\hline
\end{tabular}

Así por los coeficientes de correlación entre la Escala B-O y el CI encontramos validez concurrente, manteniendo su distancia, si bien los coeficientes son significativos pero su intensidad no pasa al $0.60(0.57)$, por lo que se puede afirmar que ambos tests miden funciones cognitivas ejecutivas manteniendo la distancia correspondiente aún en sujetos con retardo mental.

En el presente estudio hemos revisado por el método de la consistencia interna el comportamiento de la Escala B-O en sujetos con retardo mental leve y moderado, confirmándose así que la construcción de la Imagen Mental Estática está constituida por las pruebas I, II y VII y que la Imagen Mental Cinética por las pruebas III, IV, $\mathrm{V}$ y VI, encontrando correlaciones muy altas entre las pruebas correspondientes con los subtotales de cada una: 0.84 para Estáticas y 0.95 para Cinéticas. (Véase la Tabla 1.)

\section{RESULTADOS}

Inteligencia Ejecutiva en Sujetos con Retardo Mental según el Sexo y Edad Cronológica

Iniciamos el análisis partiendo del supuesto que en la condición de retardo mental leve y moderado, la edad cronológica también aquí debería plantear diferencias significativas así como por el sexo en los factores (las 7 Pruebas del B-O). Para demostrar el mencionado supuesto, con los datos obtenidos hemos contrastado 
por cada uno de los factores, primero para el grupo en general y luego por sexo y edad, para lo cual hemos utilizado el análisis de varianza, como se ve en las siguientes tablas:

Tabla 6. Promedios Aritméticos y Varianzas de los Totales en las 7 Pruebas según la Edad y el Sexo de los Sujetos

\begin{tabular}{cccccc}
\hline Edad & Grupos & Cuenta & Suma & Promedio & Varianza \\
\hline \multirow{2}{*}{12} & $\mathrm{H}$ & 6 & 177.0 & 29.50 & 261.80 \\
\cline { 2 - 6 } & $\mathrm{M}$ & 3 & 84.0 & 28.00 & 110.25 \\
\hline \multirow{2}{*}{13} & $\mathrm{H}$ & 5 & 145.0 & 29.00 & 164.75 \\
\cline { 2 - 6 } & $\mathrm{M}$ & 4 & 98.0 & 24.50 & 81.17 \\
\hline \multirow{2}{*}{14} & $\mathrm{H}$ & 5 & 176.5 & 35.30 & 208.20 \\
\cline { 2 - 5 } & $\mathrm{M}$ & 4 & 98.5 & 24.63 & 125.56 \\
\hline \multirow{2}{*}{15} & $\mathrm{H}$ & 4 & 112.5 & 28.13 & 1.23 \\
\cline { 2 - 5 } & $\mathrm{M}$ & 4 & 101.5 & 25.38 & 48.73 \\
\hline \multirow{2}{*}{16} & $\mathrm{H}$ & 6 & 181.5 & 30.25 & 255.28 \\
\cline { 2 - 5 } & $\mathrm{M}$ & 6 & 152.5 & 25.42 & 75.44 \\
\hline \multirow{2}{*}{17} & $\mathrm{H}$ & 4 & 129.5 & 32.38 & 308.73 \\
\cline { 2 - 5 } & $\mathrm{M}$ & 5 & 166.5 & 33.30 & 96.08 \\
\hline \multirow{2}{*}{18} & $\mathrm{H}$ & 4 & 148.5 & 37.13 & 71.73 \\
\cline { 2 - 5 } & $\mathrm{M}$ & 4 & 105.5 & 26.38 & 431.56 \\
\hline \multirow{2}{*}{19} & $\mathrm{H}$ & 3 & 115.0 & 38.33 & 132.50 \\
\cline { 2 - 5 } & $\mathrm{M}$ & 5 & 180.0 & 36.00 & 132.50 \\
\hline \multirow{2}{*}{20} & $\mathrm{H}$ & 3 & 115.5 & 38.50 & 99.75 \\
\cline { 2 - 5 } & $\mathrm{M}$ & 5 & 144.5 & 28.90 & 117.80 \\
\hline \multirow{3}{*}{} & Totales & 80 & 2432.0 & 30.40 & \\
\cline { 2 - 4 } & & & & &
\end{tabular}

Tabla 7 Análisis de Varianza del Total en las 7 Pruebas según la Edad y el Sexo

\begin{tabular}{ccccccc}
\hline $\begin{array}{c}\text { Origen } \\
\text { de las } \\
\text { Variaciones }\end{array}$ & $\begin{array}{c}\text { Suma } \\
\text { de } \\
\text { Cuadrados }\end{array}$ & $\begin{array}{c}\text { Grados de } \\
\text { Libertad }\end{array}$ & $\begin{array}{c}\text { Promedio } \\
\text { de los } \\
\text { Cuadrados }\end{array}$ & F & Probabilidad & $\begin{array}{c}\text { Valor } \\
\text { Critico } \\
\text { para F }\end{array}$ \\
\hline $\begin{array}{c}\text { Entre } \\
\text { Grupos }\end{array}$ & 1552.53 & 17 & 91.33 & 0.56 & 0.91 & 1.79 \\
$\begin{array}{c}\text { Dentro de } \\
\text { los Grupos }\end{array}$ & 10134.18 & 62 & 163.45 & & & \\
Total & 11686.70 & 79 & & & & \\
Sexo & 361.25 & 1 & 361.25 & 2.21 & & \\
Edad & 748.55 & 8 & 93.57 & 0.57 & & \\
Interacción & 442.73 & 8 & 55.34 & 0.34 & & \\
\hline
\end{tabular}


Tabla 8 Valores Fisher (F) por factor y total en Sujetos con Retardo Mental

\begin{tabular}{cccc}
\hline Factor & F General & F Sexo & F Edad \\
\hline P1 & 0.61 & 2.03 & 0.59 \\
P2 & 1.40 & 0.49 & 0.51 \\
P3 & 0.81 & 0.00 & 0.87 \\
P4 & 1.09 & 0.55 & 1.02 \\
P5 & 0.86 & $6.62^{*}$ & 0.63 \\
P6 & 1.55 & $3.68^{*}$ & 2.03 \\
P7 & 0.91 & $5.90^{*}$ & 0.72 \\
P Total & 0.56 & 2.21 & 0.57 \\
\hline
\end{tabular}

$\left.{ }^{*}\right) \mathrm{p}<0.05$

De la tabla 4 se desprende, que en ninguno de los factores o Pruebas de la Escala, hay diferencias significativas cuando se efectúa el análisis con el grupo total, hecho que indica que los resultados son similares en el continum cronológico.

Para confirmar si efectivamente los resultados son similares sin variaciones significativas, contrastamos los puntajes obtenidos para cada edad usando la razón Fisher $(\mathrm{F})$ o análisis de varianza, confirmando efectivamente que la edad cronológica no plantea diferencias.

Analizando las diferencias según el sexo en los siete factores, hemos encontrado en tres factores diferencias significativas que representan el $43 \%$, en tanto que en el 57\% de comparaciones no se registra diferencias, contrastando estos porcentajes encontramos un valor Chi-cuadrada $\left(\mathrm{x}^{2}\right)$ de 0.16 que indica que el porcentaje de factores que difieren según el sexo comparado con los factores que no difieren no es relevante significativamente, por tanto las diferencias encontradas son productos de variaciones importantes pero que no llegan a marcar direccionalidad típica a favor de los varones, sólo habría tendencia no significativa; tal como puede verse en la siguiente tabla.

Tabla 9 Prueba Chi- cuadrada con Corrección de Yates y Fórmula Abreviada

\begin{tabular}{c|c|c|c}
\cline { 2 - 3 }$f 0 \%$ & $57 \%$ & $43 \%$ & $100 \%$ \\
\hline$f \mathrm{e} \%$ & $50 \%$ & $50 \%$ & $100 \%$ \\
& $\downarrow$ & $\downarrow$ & \\
$\left(f_{0}-f_{\mathrm{e}}\right)$ & $7 \%$ & $7 \%$ & \\
Corrección $(-5 \%)$ & $2 \%$ & $2 \%$ & \\
$\left(f_{0}-f_{\mathrm{e}}\right)^{2}$ & $4 \%$ & $4 \%$ & \\
$\mathrm{x}^{2} \%=2\left(f_{0}-f_{\mathrm{e}}\right)^{2}$ & $=2(4 \%)$ & $=0.16$ & \\
$f_{\mathrm{e}}$ & 50 &
\end{tabular}




\section{La Imagen Mental Estática según el Retardo Mental}

La imagen mental estática es la resultante de la forma como el examinado procesa sus respuestas a la Pruebas 1, 2, y 7 de la Escala B-O. Al analizar el sexo y el rendimiento en las pruebas de la Imagen Mental Estática, tenemos dos subgrupos de sujetos en función a la categoría intelectual y para cada grupo dos subgrupos según el sexo, que nos permite contrastar cada uno de los componentes mediante el análisis de varianza de dos factores y dos vías por factor:

En la Prueba I hemos encontrado que el género no plantea diferencias como sí la categoría intelectual tal como se puede ver en las siguientes tablas:

Tabla 10. Promedios Aritméticos y Varianzas según el Tipo de Retardo y Sexo de los Sujetos en la Prueba I

\begin{tabular}{cccccc}
\hline & Grupos & Cuenta & Suma & Promedio & Varianza \\
\hline \multirow{2}{*}{ RML } & Hombres & 19 & 139.5 & 7.34 & 15.36 \\
\cline { 2 - 6 } & Mujeres & 20 & 107.5 & 5.38 & 8.55 \\
\hline \multirow{2}{*}{ RMM } & Hombres & 21 & 105.5 & 5.02 & 10.01 \\
\cline { 2 - 6 } & Mujeres & 20 & 94.0 & 4.70 & 6.46 \\
\hline & Totales & 80 & 446.5 & 5.58 & \\
\hline
\end{tabular}

Tabla 11. Análisis de Varianza de la Prueba I en general, según el Tipo de Retardo y Sexo

\begin{tabular}{ccccccc}
\hline $\begin{array}{c}\text { Origen de las } \\
\text { Variaciones }\end{array}$ & $\begin{array}{c}\text { Suma } \\
\text { de } \\
\text { Cuadrados }\end{array}$ & $\begin{array}{c}\text { Grados de } \\
\text { Libertad }\end{array}$ & $\begin{array}{c}\text { Promedio } \\
\text { de los } \\
\text { Cuadrados }\end{array}$ & F & Probabilidad & $\begin{array}{c}\text { Valor } \\
\text { Crítico } \\
\text { para F }\end{array}$ \\
\hline $\begin{array}{c}\text { Entre } \\
\text { Grupos }\end{array}$ & 81.82 & 3 & 27.27 & 2.72 & 0.05 & 2.72 \\
$\begin{array}{c}\text { Dentro de los } \\
\text { grupos }\end{array}$ & 761.90 & 76 & 10.03 & & & \\
$\quad$ Total & 843.72 & 79 & & & & \\
Inteligencia & 43.04 & 1 & 43.04 & 4.29 & 0.05 & 3.96 \\
$\quad$ Sexo & 23.65 & 1 & 23.65 & 2.36 & 0.08 & 3.96 \\
Interacción & 15.12 & 1 & 15.12 & 1.51 & 0.11 & 3.96 \\
\hline
\end{tabular}

De la tabla se desprende que la diferencia encontrada en general se debe a la categoría intelectual y no al sexo.

En la Prueba II hemos efectuado análisis similar y hemos encontrado que el tipo de retardo y el sexo actúan sin producir variabilidad significativa tal como puede verse en las siguientes tablas: 
Tabla 12. Promedios Aritméticos y Varianzas según el Tipo de Retardo y Sexo de los Sujetos en la Prueba II

\begin{tabular}{cccccc}
\hline & Grupos & Cuenta & Suma & Promedio & Varianza \\
\hline \multirow{2}{*}{ RML } & Hombres & 19 & 80.0 & 4.21 & 1.62 \\
\cline { 2 - 6 } & Mujeres & 20 & 91.0 & 4.55 & 0.68 \\
\hline \multirow{2}{*}{ RMM } & Hombres & 21 & 84.0 & 4.00 & 1.60 \\
\cline { 2 - 6 } & Mujeres & 20 & 81.0 & 4.05 & 1.73 \\
\hline & Totales & 80 & 336.0 & 4.20 & \\
\hline
\end{tabular}

Tabla 13. Análisis de Varianza de la Prueba II en general, según el Tipo de Retardo y Sexo

\begin{tabular}{ccccccc}
\hline $\begin{array}{c}\text { Origen } \\
\text { de las } \\
\text { Variaciones }\end{array}$ & $\begin{array}{c}\text { Suma } \\
\text { de Cuadrados }\end{array}$ & $\begin{array}{c}\text { Grados de } \\
\text { Libertad }\end{array}$ & $\begin{array}{c}\text { Promedio } \\
\text { de los } \\
\text { Cuadrados }\end{array}$ & F & Probabilidad & $\begin{array}{c}\text { Valor } \\
\text { Crítico } \\
\text { para F }\end{array}$ \\
\hline $\begin{array}{c}\text { Entre } \\
\text { Grupos }\end{array}$ & 3.74 & 3 & 1.25 & 0.89 & 0.45 & 2.72 \\
$\begin{array}{c}\text { Dentro de los } \\
\text { grupos }\end{array}$ & 107.06 & 76 & 1.41 & & & \\
Total & 110.80 & 79 & & & & \\
$\begin{array}{c}\text { Inteligencia } \\
\text { Sexo }\end{array}$ & 2.59 & 1 & 2.59 & 1.84 & 0.10 & 3.96 \\
Interacción & 0.80 & 1 & 0.80 & 0.57 & 0.14 & 3.96 \\
\hline
\end{tabular}

En lo concerniente a la Prueba VII que es el otro indicador de la imagen mental estática, hemos encontrado, que el determinante de la diferencia es el tipo de retardo, el sexo no tiene ninguna incidencia como puede verse en las siguientes tablas:

Tabla 14. Promedios Aritméticos y Varianzas según el Tipo de Retardo y Sexo de los Sujetos en la Prueba VII

\begin{tabular}{cccccc}
\hline & Grupos & Cuenta & Suma & Promedio & Varianza \\
\hline \multirow{2}{*}{ RML } & Hombres & 19 & 100.5 & 5.29 & 3.59 \\
\cline { 2 - 6 } & Mujeres & 20 & 61.5 & 3.08 & 1.30 \\
\hline \multirow{2}{*}{ RMM } & Hombres & 21 & 73.0 & 3.48 & 3.69 \\
\cline { 2 - 6 } & Mujeres & 20 & 69.0 & 3.45 & 4.39 \\
\hline & Totales & 80 & 304.0 & 3.80 & \\
\hline
\end{tabular}


Tabla 15. Análisis de Varianza de la Prueba VII en general, según el Tipo de Retardo y Sexo

\begin{tabular}{ccccccc}
\hline $\begin{array}{c}\text { Origen } \\
\text { de las } \\
\text { Variaciones }\end{array}$ & $\begin{array}{c}\text { Suma } \\
\text { de } \\
\text { Cuadrados }\end{array}$ & $\begin{array}{c}\text { Grados } \\
\text { de } \\
\text { Libertad }\end{array}$ & $\begin{array}{c}\text { Promedio } \\
\text { de los } \\
\text { Cuadrados }\end{array}$ & F & Probabilidad & $\begin{array}{c}\text { Valor } \\
\text { Critico } \\
\text { para F }\end{array}$ \\
\hline $\begin{array}{c}\text { Entre } \\
\text { Grupos }\end{array}$ & 57.32 & 3 & 19.11 & 5.89 & 0.00 & 4.61 \\
$\begin{array}{c}\text { Dentro de los } \\
\text { grupos }\end{array}$ & 246.48 & 76 & 3.24 & & & \\
Total & 303.80 & 79 & & & & \\
$\begin{array}{c}\text { Inteligencia } \\
\text { Sexo }\end{array}$ & 9.53 & 1 & 9.53 & 2.94 & 0.01 & 3.46 \\
Interacción & 23.11 & 1 & 23.11 & 7.13 & 0.09 & 3.46 \\
\hline
\end{tabular}

Finalmente para demostrar nuestra tesis principal hemos contrastado las pruebas que constituyen la imagen mental estática según la inteligencia ya que el sexo no es una variable que actúe sobre ésta, como se podrá ver las medias aritméticas son mayores en el grupo con retardo mental leve, aún cuando sólo es significativa en la Prueba I, hecho que ha determinado que la imagen mental estática resulte significativamente mayor en el grupo con retardo mental leve, tal como puede verse en la siguiente tabla:

Tabla 16. Diferencia de Medias por Pruebas en Imagen Mental Estática por Tipo de Retardo

\begin{tabular}{|c|c|c|c|c|c|c|c|c|c|c|}
\hline & \multicolumn{3}{|c|}{ RML } & \multicolumn{2}{|r|}{ RMM } & \multirow[b]{2}{*}{ DS2 } & \multirow[b]{2}{*}{ Diferencia } & \multirow[b]{2}{*}{$\mathrm{EE}$} & \multirow[b]{2}{*}{$\mathrm{t}$} & \multirow[b]{2}{*}{$\mathrm{P}$} \\
\hline & $\mathrm{N}$ & Media & DS2 & $\mathrm{n}$ & Media & & & & & \\
\hline $\mathrm{P} 1$ & 39 & 6.33 & 12.54 & 41 & 4.87 & 8.10 & 1.47 & 0.72 & 2.04 & 0.05 \\
\hline $\mathrm{P} 2$ & 39 & 4.38 & 1.14 & 41 & 4.02 & 1.62 & 0.36 & 0.26 & 1.37 & 0.07 \\
\hline P7 & 39 & 4.15 & 3.61 & 41 & 3.46 & 3.93 & 0.69 & 0.43 & 1.59 & 0.08 \\
\hline $\begin{array}{c}\text { I.M. } \\
\text { Estática }\end{array}$ & 39 & 14.87 & 21.31 & 41 & 12.35 & 27.30 & 2.52 & 1.10 & 2.29 & 0.05 \\
\hline
\end{tabular}

\section{La Imagen Mental Cinética según el Retardo Mental}

La imagen mental cinética en la Prueba B-O está constituida por las Pruebas $3,4,5$ y 6 , que analizaremos primero cada uno por separado porque creemos importante estudiar si el sexo es una variable que plantea diferencias o si la variable importante es la inteligencia para lo cual hemos efectuado el mismo análisis del acápite anterior, habiendo encontrado: 
En la Prueba III, el sexo no plantea diferencias significativas como sí ocurre con la inteligencia, para la cual encontrarnos una razón F de 10.27 muy significativa aún para el 0.01 de error, como se puede ver en las siguientes tablas.

Tabla 17. Promedios Aritméticos y Varianzas según el Tipo de Retardo y Sexo de los Sujetos en la Prueba III

\begin{tabular}{cccccc}
\hline & Grupos & Cuenta & Suma & Promedio & Varianza \\
\hline \multirow{2}{*}{ RML } & Hombres & 19 & 146.0 & 7.68 & 7.45 \\
\cline { 2 - 6 } & Mujeres & 20 & 137.0 & 6.85 & 8.24 \\
\hline \multirow{2}{*}{ RMM } & Hombres & 21 & 103.0 & 4.90 & 7.59 \\
\cline { 2 - 6 } & Mujeres & 20 & 112.0 & 5.60 & 8.25 \\
\hline \multirow{2}{*}{} & Totales & 80 & 498.0 & 6.23 & \\
\hline
\end{tabular}

Tabla 18. Análisis de Varianza de la Prueba III en general, según el Tipo de Retardo y Sexo

\begin{tabular}{ccccccc}
\hline $\begin{array}{c}\text { Origen } \\
\text { de las } \\
\text { variaciones }\end{array}$ & $\begin{array}{c}\text { Suma } \\
\text { de } \\
\text { cuadrados }\end{array}$ & $\begin{array}{c}\text { Grados de } \\
\text { Libertad }\end{array}$ & $\begin{array}{c}\text { Promedio } \\
\text { de los } \\
\text { Cuadrados }\end{array}$ & F & Probabilidad & $\begin{array}{c}\text { Valor } \\
\text { Crítico } \\
\text { para F }\end{array}$ \\
\hline $\begin{array}{c}\text { Entre } \\
\text { grupos }\end{array}$ & 92.69 & 3 & 30.90 & 3.92 & 0.01 & 2.72 \\
$\begin{array}{c}\text { Dentro de los } \\
\text { grupos }\end{array}$ & 599.26 & 76 & 7.89 & & & \\
$\quad$ Total & 691.95 & 79 & & & & \\
Inteligencia & 80.95 & 1 & 80.95 & 10.27 & 0.01 & 3.96 \\
$\quad$ Sexo & 0.00 & 1 & 0.00 & 0.00 & 0.99 & 3.96 \\
Interacción & 11.73 & 1 & 11.73 & 1.49 & 0.15 & 3.96 \\
\hline
\end{tabular}

En la Prueba IV los resultados son similares en los cuatro subgrupos lo que significa que la condición intelectual requerida para la realización de esta tarea es independiente del sexo y de la inteligencia general, ya que los valores de los promedios no difieren significativamente, tal como se puede ver en las siguientes tablas:

Tabla 19. Promedios Aritméticos y Varianzas según el Tipo de Retardo y Sexo de los Sujetos en la Prueba IV

\begin{tabular}{cccccc}
\hline & Grupos & Cuenta & Suma & Promedio & Varianza \\
\hline \multirow{2}{*}{ RML } & Hombres & 19 & 68.0 & 3.58 & 9.59 \\
\cline { 2 - 6 } & Mujeres & 20 & 73.0 & 3.65 & 5.82 \\
\hline \multirow{2}{*}{ RMM } & Hombres & 21 & 53.0 & 2.52 & 7.06 \\
\cline { 2 - 6 } & Mujeres & 20 & 66.0 & 3.30 & 8.22 \\
\hline & Totales & 80 & 260.0 & 3.25 & \\
\hline
\end{tabular}


Tabla 20. Análisis de Varianza de la Prueba IV en general, según el Tipo de Retardo y Sexo

\begin{tabular}{ccccccc}
\hline $\begin{array}{c}\text { Origen } \\
\text { de las } \\
\text { variaciones }\end{array}$ & $\begin{array}{c}\text { Suma } \\
\text { de } \\
\text { cuadrados }\end{array}$ & $\begin{array}{c}\text { Grados } \\
\text { de } \\
\text { Libertad }\end{array}$ & $\begin{array}{c}\text { Promedio } \\
\text { de los } \\
\text { Cuadrados }\end{array}$ & F & Probabilidad & $\begin{array}{c}\text { Valor } \\
\text { Crítico } \\
\text { para F }\end{array}$ \\
\hline $\begin{array}{c}\text { Entre } \\
\text { Grupos }\end{array}$ & 16.38 & 3 & 5.46 & 0.71 & 0.55 & 2.72 \\
$\begin{array}{c}\text { Dentro de los } \\
\text { grupos }\end{array}$ & 580.62 & 76 & 7.64 & & & \\
Total & 597.00 & 79 & & & & \\
Inteligencia & 10.16 & 1 & 10.16 & 1.33 & 0.01 & 3.96 \\
Sexo & 4.05 & 1 & 4.05 & 0.53 & 0.44 & 3.96 \\
Interacción & 2.17 & 1 & 2.17 & 0.28 & 0.08 & 3.96 \\
\hline
\end{tabular}

En la Prueba V, el género plantea diferencia siendo mayor el rendimiento en los hombres, también la inteligencia plantea diferencia a favor de la categoría retardo mental leve, como se puede leer en las siguientes tablas:

Tabla 21. Promedios Aritméticos y Varianzas según el Tipo de Retardo y Sexo de los Sujetos en la Prueba V

\begin{tabular}{cccccc}
\hline & Grupos & Cuenta & Suma & Promedio & Varianza \\
\hline \multirow{2}{*}{ RML } & Hombres & 19 & 108.0 & 5.68 & 8.78 \\
\cline { 2 - 6 } & Mujeres & 20 & 73.0 & 3.65 & 5.29 \\
\hline \multirow{2}{*}{ RMM } & Hombres & 21 & 78.0 & 3.71 & 5.51 \\
\cline { 2 - 6 } & Mujeres & 20 & 49.0 & 2.45 & 6.26 \\
\hline \multicolumn{2}{c}{ Totales } & 80 & 308.0 & 3.85 & \\
\hline
\end{tabular}

Tabla 22. Análisis de Varianza de la Prueba V en general, según el Tipo de Retardo y Sexo

\begin{tabular}{c|c|c|c|c|c|c}
\hline $\begin{array}{c}\text { Origen } \\
\text { de las }\end{array}$ & $\begin{array}{c}\text { Suma } \\
\text { de } \\
\text { Variaciones }\end{array}$ & \multicolumn{1}{c}{$\begin{array}{c}\text { Grados de } \\
\text { Cuadrados }\end{array}$} & $\begin{array}{c}\text { Pibertad } \\
\text { Promedio } \\
\text { de los } \\
\text { Cuadrados }\end{array}$ & \multicolumn{2}{c}{ F } & \multicolumn{2}{c}{ Probabilidad } & $\begin{array}{c}\text { Valor } \\
\text { Crítico } \\
\text { para F }\end{array}$ \\
\hline $\begin{array}{c}\text { Entre grupos } \\
\text { Dentro de los }\end{array}$ & 104.31 & 3 & 34.77 & 5.42 & 0.00 & 2.49 \\
$\quad 487.89$ & 76 & 6.42 & & & \\
grupos & 592.20 & 79 & & & & \\
$\quad$ Total & 47.62 & 1 & 47.62 & 7.42 & 0.01 & 3.96 \\
Inteligencia & 51.20 & 1 & 51.20 & 7.98 & 0.01 & 3.96 \\
$\quad$ Sexo & 1 & 5.49 & 0.86 & 0.15 & 3.96 \\
\hline Interacción & 5.49 & & & & & \\
\hline
\end{tabular}


En la Prueba VI, el proceso mental requerido, no difiere según el género, aún cuando los puntajes son mayores en los hombres sin llegar a niveles significativos. En lo que respecta a la inteligencia hemos encontrado diferencias significativas en dirección favorable a la categoría de retardo mental leve, tal como puede verse en las siguientes tablas.

Tabla 23. Promedios Aritméticos y Varianzas según el Tipo de Retardo y Sexo de los Sujetos en la Prueba VI

\begin{tabular}{cccccc}
\hline & Grupos & Cuenta & Suma & Promedio & Varianza \\
\hline \multirow{2}{*}{ RML } & Hombres & 19 & 94.5 & 4.97 & 9.46 \\
\cline { 2 - 6 } & Mujeres & 20 & 78.0 & 3.90 & 5.31 \\
\hline \multirow{2}{*}{ RMM } & Hombres & 21 & 68.0 & 3.24 & 8.27 \\
\cline { 2 - 6 } & Mujeres & 20 & 40.0 & 2.00 & 3.50 \\
\hline \multicolumn{2}{c}{ Totales } & 80 & 280.5 & 3.51 & \\
\hline
\end{tabular}

Tabla 24. Análisis de Varianza de la Prueba VI en general, según el Tipo de Retardo y Sexo

\begin{tabular}{ccccccc}
\hline $\begin{array}{c}\text { Origen } \\
\text { de las } \\
\text { Variaciones }\end{array}$ & $\begin{array}{c}\text { Suma } \\
\text { de } \\
\text { Cuadrados }\end{array}$ & $\begin{array}{c}\text { Grados } \\
\text { de } \\
\text { libertad }\end{array}$ & $\begin{array}{c}\text { Promedio } \\
\text { de los } \\
\text { Cuadrados }\end{array}$ & F & Probabilidad & $\begin{array}{c}\text { Valor } \\
\text { Crítico } \\
\text { para F }\end{array}$ \\
\hline $\begin{array}{c}\text { Entre grupos } \\
\text { Dentro de los }\end{array}$ & 90.9 & 3 & 30.30 & 4.58 & 0.01 & 2.7 \\
grupos & 502.85 & 76 & 6.62 & & & \\
$\quad$ Total & 593.75 & 79 & & & & \\
Inteligencia & 63.97 & 1 & 63.97 & 9.67 & 0.01 & 3.96 \\
$\quad$ Sexo & 24.75 & 1 & 24.75 & 3.74 & 0.07 & 3.96 \\
Interacción & 2.18 & 1 & 2.18 & 0.33 & 0.14 & 3.96 \\
\hline
\end{tabular}

Finalmente en vista que el sexo no es una variable que plantee diferencias como sí lo es la inteligencia hemos contrastado los valores promedio de cada uno de las pruebas que componen la Imagen Mental Cinética, encontrando que este tipo de imagen mental requiere de mayor inteligencia, afirmación que se desprende de las diferencias significativas encontradas y que puede verse en la siguiente tabla:

Tabla 25. Diferencia de medias por Pruebas en Imagen Mental Cinética por Tipo de Retardo

\begin{tabular}{|c|c|c|c|c|c|c|c|c|c|c|}
\hline & \multicolumn{3}{|c|}{ RML } & \multicolumn{3}{|c|}{ RMM } & \multirow[b]{2}{*}{ Diferencia } & \multirow[b]{2}{*}{$\mathrm{EE}$} & \multirow[b]{2}{*}{$\mathrm{t}$} & \multirow[b]{2}{*}{$\mathrm{p}$} \\
\hline & $\mathrm{N}$ & Media & DS2 & $\mathrm{n}$ & Media & DS2 & & & & \\
\hline P3 & 39 & 7.26 & 7.83 & 41 & 5.24 & 7.84 & 2.01 & 0.63 & 3.21 & 0.01 \\
\hline P4 & 39 & 3.62 & 7.45 & 41 & 2.90 & 7.59 & 0.71 & 0.61 & 1.16 & 0.08 \\
\hline P5 & 39 & 4.64 & 7.87 & 41 & 3.10 & 6.14 & 1.54 & 0.59 & 2.60 & 0.01 \\
\hline P6 & 39 & 4.42 & 7.43 & 41 & 2.63 & 6.19 & 1.79 & 0.58 & 3.06 & 0.01 \\
\hline $\begin{array}{c}\text { I.M. } \\
\text { Cinética }\end{array}$ & 39 & 19.94 & 70.24 & 41 & 13.88 & 53.01 & 6.06 & 1.76 & 3.44 & 0.00 \\
\hline
\end{tabular}




\section{DISCUSIÓN}

Aceptamos la hipótesis nula de la hipótesis de la discriminación genética de la Escala Borelli-Oléron, cuya demostración por Análisis de Varianza (Tabla 3) y Chi-Cuadrada (Tabla 5) indica que no existen diferencias significativas en la variable medida, es decir en la inteligencia ejecutiva ni por la edad ni por el sexo de la muestra.

Aceptamos la hipótesis del Dimensionamiento de la Escala Borelli-Oléron, cuya demostración por la Matriz R (Tabla 1), indica que las variables medidas están positiva y altamente correlacionadas en torno a la dimensión Estática y Cinética de la Imagen Mental Reproductora de acuerdo a la construcción realizada por nosotros para esta investigación según el Análisis de Contenido en las pruebas de la Escala (Tabla 4).

Aceptamos la hipótesis de la Imagen Mental Reproductora Vs. Retardo Mental, cuya demostración por Análisis de Varianza para Estáticas (Tablas 7, 9, 11) y para Cinéticas (Tablas 14, 16, 18, 20) y su respectiva Diferencia de Medias (Tablas 12 y 21), indica que las variables medidas, es decir las Imágenes Mentales Estáticas y Cinéticas difieren significativamente entre el Retardo Mental Leve y Moderado siendo esta diferencia a favor del Retardo Mental Leve en las estáticas $(p=0.05)$ $\mathrm{y}$, más aún, en las cinéticas $(\mathrm{p}=0.00)$.

De esta manera se alcanzó a mostrar que la imagen mental está implicada como una función cognitiva figural de la inteligencia ejecutiva, mediante los constructos de imagen mental estática y cinética, en las Pruebas de la Escala B-O, en el retardo mental leve y moderado.

\section{CONCLUSIONES}

1. La Escala Borelli-Oléron como indicador de Inteligencia Ejecutiva, no produce discriminación genética (diferencia significativa por sexo y por edad cronológica) en la muestra de sujetos con Retardo Mental.

2. Las Pruebas I, II y VII de la Escala Borelli-Oléron correlacionan positivamente elevada con la Imagen Mental Reproductora Estática en los sujetos con Retardo Mental.

3. Las Pruebas III, IV, V y VI de la Escala Borelli-Oléron correlacionan positivamente elevada con la Imagen Mental Reproductora Cinética en los sujetos con Retardo Mental.

4. El rendimiento en la dimensión estática de la Escala Borelli-Oléron en los sujetos con Retardo Mental Leve es significativamente mayor que en los Retardo Mental Moderado.

5. El rendimiento en la dimensión cinética de la Escala Borelli-Oléron en los sujetos con Retardo Mental Leve es significativamente mayor que en los Retardo Mental Moderado.

6. Los sujetos con Retardo Mental Leve presentan mayor rendimiento tanto en la dimensión estática como en la cinética que los sujetos con Retardo Mental Moderado pero la diferencia es más significativa en la cinética. 


\section{REFERENCIAS BIBLIOGRÁFICAS}

Borelli, M. y Oléron, P. (1995). Escala ejecutiva de Borelli-Oléron. (Trad. J.C. Rivera B.). En Informe de Investigación (Código 951801011). Manuscrito inédito, Instituto de Investigaciones de la Facultad de Psicología, UNMSM, Lima. (Original en francés, 1964).

Inhelder, B. (1971). El Diagnóstico del Razonamiento en los Débiles Mentales. (Trad. A. Leal). Barcelona: Nova Terra. (Original en francés, 1963).

Laurendeau, M. y Pinard, A. (1976). Las Primeras Nociones Espaciales en el Niño: Examen de la hipótesis de Jean Piaget. (Vol. I). (Trad. R. Solis). Buenos Aires: Glem. (Original en francés, 1968).

Magnusson, D. (1983). Teoría de los Test. (Trad. J. Aguilar). México: Trillas. (Original en inglés, 1967).

Pain, S. (1974). Psicometría genética. (2 Ed.). Buenos Aires: Nueva Visión.

Piaget, J. (1961). La formación del símbolo en el niño. (Trad. J. Gutiérrez). México DF. México: Fondo de Cultura Económica. (Original en francés, 1959).

Piaget, J. (1975). Les Mécanismes Perceptifs. [Los mecanismos perceptivos] (2 Ed.). Paris: Presses Universitaires de France.

Piaget, J. et Inhelder, B. (1966). L'image mental chez l'enfant. [La imagen mental en el niño]. Paris: Presses Universitaires de France.

Piaget, J. et Inhelder, B. (1972). La représentation de l'espace chez l'enfant. [La representación del espacio en el niño] ( $2^{\circ}$ Ed.). Paris: Presses Universitaires de France.

Piaget, J. e Inhelder, B. (1973). Las imágenes mentales. En Piaget, J. y Fraisse, P. (Comp.). La inteligencia. Tratado de Psicología Experimental. (Pp. 87-141). (Vol.7). (Trad. V. Fischman). Buenos Aires: Paidós. (Original en francés, 1967).

Pinol-Douriez, M. (1979). La Construcción del Espacio en el Niño: El desarrollo semiótico del esquema corporal. (Trad. S. Scialdone). Madrid: Pablo del Río-Editor. (Original en francés).

Rivera, J. C. (1995). Imagen mental en niños con retardo mental. Informe de investigación (Código 951801011). Manuscrito inédito, Instituto de Investigaciones de la Facultad de Psicología, UNMSM, Lima-Perú.

Tavella, N. M. (1966). Características de estructura y características de los tests. En Székely, B. Los Tests. Manual de Técnicas de Exploración Psicológica. (Pp. 109152). Vol. I. (5 Ed.). Buenos Aires: Kapelusz. 